\title{
IMPORTANCIA DE LOS REFERENCIADORES BIBLIOGRÁFICOS EN LA GESTIÓN DE LA INFORMACIÓN CIENTÍFICA EN TESISTAS UNIVERSITARIOS
}

\author{
Lloy Pinedo-Tuanama* \\ Facultad de Ingeniería de Sistemas e Informática. Universidad Nacional de San Martín - Tarapoto. \\ Miguel Valles-Coral** \\ Facultad de Ingeniería de Sistemas e Informática. Universidad Nacional de San Martín - Tarapoto.
}

\begin{abstract}
Resumen: Gestionar la información científica en el proceso investigativo es un gran desafío para los tesistas universitarios debido a la dificultad de sistematizar y estructurar el exceso de información disponible en diferentes fuentes digitales o físicas. El objetivo del estudio fue analizar la importancia de los referenciadores bibliográficos como herramienta tecnológica para la gestión de la información científica en tesistas universitarios. Se llevó a cabo una revisión bibliográfica sistemática realizando búsquedas en bases de datos electrónicas de artículos científicos publicados en revistas indexadas a Latindex Catálogo, Scielo y Scopus entre los años 2016 y 2020 . Los resultados de la revisión describen las características, funcionalidades, aspectos y estudios principales sobre los beneficios que brindan los referenciadores bibliográficos para gestionar la información científica. Concluimos que funciones como la organización de la información, citación y referenciación bibliográfica y colaboración entre investigadores proporcionados por los referenciadores bibliográficos, constituyen recursos importantes para los tesistas universitarios.
\end{abstract}

Palabras clave: Calidad; citas; gestión; información; investigación; referencias; referenciadores bibliográficos.

\begin{abstract}
Title: IMPORTANCE OF REFERENCE MANAGER IN THE MANAGEMENT OF SCIENTIFIC INFORMATION IN UNIVERSITY THESES STUDENTS.

Abstract: Managing scientific information in the research process is a great challenge for university thesis students due to the difficulty of systematizing and structuring the excess of information available in different digital or physical sources. The objective of the study was to analyze the importance of reference manager as a technological tool for the management of scientific information in university theses students. We carried out a systematic bibliographic review by searching electronic databases of scientific articles published in journals indexed to Latindex Catalog, Scielo and Scopus between the years 2016 and 2020. The results of the review describe the characteristics, functionalities, aspects and studies main ones on the benefits that reference manager provide to manage scientific information. We conclude that functions such as the organization of information, bibliographic citation and referencing, and collaboration between researchers provided by reference manager, constitute important resources for university thesis students.
\end{abstract}

Keywords: Citations; information; management; quality; reference manager; references; research.

Copyright: (C) 2021 Servicio de Publicaciones de la Universidad de Murcia (Spain). Este es un artículo de acceso abierto distribuido bajo los términos de la licencia Creative Commons Reconocimiento 4.0 Internacional (CC BY 4.0).

\section{INTRODUCCIÓN}

La gestión de la información en investigación comprende conocimientos técnicos y metodológicos necesarios en el proceso investigativo que se consideran esenciales en el aprendizaje académico a partir de la identificación de intereses en temas de investigación científica (Rojas Betancur y Méndez Villamizar, 2017; Medina Martínez et al., 2020); este proceso formativo, según Cayo-Rojas y Agramonte-Rosell (2020), constituye la parte esencial de la misión y visión de las universidades, por lo que se requiere la inclusión estratégica de competencias conceptuales, procedimentales y actitudinales en la formación investigativa de los tesistas.

Dicha actividad, según Rivera Obregón et al. (2018) se relaciona con la construcción del conocimiento e involucra la gestión de competencias para elaborar la revisión literaria, organizar la información, administrar citas y gestionar referencias bibliográficas, impactando directamente en el desempeño académico y en los resultados de las investigaciones.

\footnotetext{
*1pinedo@unsm.edu.pe

**mavalles@unsm.edu.pe

Recibido: 25-01-2021; $2^{\mathrm{a}}$ versión: 24-04-2021; aceptado: 25-05-2021.
}

PINEDO TUANAMA, LL. y VALLES CORAL, M. Importancia de los referenciadores bibliográficos en la gestión de la información científica en tesistas universitarios. Anales de Documentación, 2021, vol. 24, $\mathrm{n}^{\mathrm{o}} 2$. Disponible en: http://dx.doi.org/10.6018/analesdoc.465091. 
Para los autores Martín y Lafuente (2017), la revisión del estado del arte es el proceso principal en el desarrollo de la investigación; ya que según Grijalva et al. (2019) permite obtener información de estudios o experimentos realizados de acuerdo a la especificidad del tema a investigar. Posteriormente, las citas y referencias bibliográficas otorgan reconocimiento a los autores de investigaciones relacionadas en dicho proceso (Cantín y Chuhuaicura, 2016); estos son elaborados según criterios bibliográficos y editoriales establecidos en los reglamentos de investigación de cada universidad.

Es preciso señalar que, la gestión de la información permite identificar el conocimiento científico con el fin de abordar el planteamiento del problema, elaborar el marco teórico y orientar el desarrollo de la metodología de la investigación (Rivera Echegaray et al., 2017); por ello Rodríguez Picornell y del Pozo Rodríguez (2019) señalan que se basa en aprendizaje sustentado en conocimiento tecnológico, necesario para la obtención y manipulación de la información.

Así, Gallegos, Peralta y Guerrero (2017) reconocen la importancia de incorporar herramientas tecnológicas en la gestión de la información científica que aporte al investigador manejo ágil y adaptable de sus necesidades, además Dal Sasso Mendes, de Campos Pereira Silveira y Galvão (2019) señalan que los referenciadores bibliográficos proporcionan recursos para la viabilidad en la gestión de la información, mejorando la calidad de las investigaciones. En esa misma perspectiva, Reyes Pérez, Cárdenas Zea y Gavilánez Buñay (2020) evidencian la necesidad de incluir su uso en el proceso investigativo, debido al desafío que enfrentan los estudiantes en gestionar la información científica, esto con la finalidad de mejorar la capacidad de entender, seleccionar, organizar, procesar y transformar la información en conocimiento.

La utilidad de los referenciadores bibliográficos, tales como Mendeley, Zotero, EndNote Basic, RefWorks entre otros, permiten gestionar la información científica a través del almacenamiento y organización de la información, la interrelación y difusión de investigaciones, y la gestión de citas y referencias, facilitando la adaptabilidad bibliográfica de investigaciones a determinados estilos estandarizados de forma automática (Fernández Izquierdo, 2018; Díez, Gullón y Fernández, 2019).

Según Antúnez Sánchez y Veytia Bucheli (2020), en Iberoamérica el gestor más utilizado es Mendeley (36,70\%), seguido de EndNote $(29,10 \%)$ y Zotero $(25,90 \%)$, evidencian además la necesidad de fortalecer la cultura del uso de herramientas tecnológicas ya que son imprescindibles en el desarrollo de trabajos de investigación y necesarios para garantizar su calidad.

Pese a la necesidad de incorporar las Tecnologías de Información y Comunicaciones (TIC) en el proceso investigativo, específicamente en la gestión de la información científica, en Perú, Valles-Coral, Riascos-Armas y Hernandez-Torres (2020) reconocen la deficiente difusión del uso de referenciadores bibliográficos en la investigación; además Milewski y Williamson (2017) indican que su desconocimiento dificulta la gestión de citas y referencias bibliográficas, impidiendo dar valor agregado a la investigación por la necesidad de realizar este proceso de manera manual.

Adicionalmente, Fryš y Kovářová (2020) refieren que las citas y referencias son elaboradas sin considerar normas o estilos estandarizados, al mismo tiempo prevalece la incongruencia entre fuentes citadas en el documento y la lista de referencias (Perdomo et al., 2020), de modo que, según López Padrón et al. (2019), los resultados de las investigaciones adolecen graves faltas editoriales en relación a la revisión del estado del arte.

Por otro lado, la deficiencia en la gestión de la información científica dificulta la publicación de artículos científicos a partir de los resultados de las investigaciones, debido a la imposibilidad de adecuarse a los criterios editoriales y bibliográficos exigidos por las revistas científicas (Atamari-Anahui, Sucasaca-Rodríguez y Marroquin-Santa Cruz, 2016; Valles-Coral, 2019).

Nuestra revisión entonces, tiene la finalidad de analizar la importancia de los referenciadores bibliográficos en la gestión de la información científica, a través de una revisión bibliográfica sistemática, en la que se recopila información relevante para que los tesistas universitarios puedan formalizar su uso de manera que garanticen la calidad bibliográfica y editorial en los resultados de las investigaciones, al mismo tiempo logren posicionarse como referentes en el campo de la investigación científica. 


\section{MATERIAL Y MÉTODO}

El reconocimiento de la información científica es base para el desarrollo de la investigación y se relaciona con la tecnología digital como principal fuente de datos en las diferentes disciplinas (Semeler y Pinto, 2020). Consolidar competencias para efectuar dicho proceso permite aprovechar la información estratégicamente para fines académicos (García Valdés, 2016). De aquí nace la importancia de gestionar, recopilar, organizar, manipular y analizar la información. Es por ello que nos preguntamos ¿Qué importancia tienen los referenciadores bibliográficos en la gestión de la información científica en tesistas universitarios?

Para responder esta pregunta, nos basamos en la metodología planteada por Riesenberg y Justice (2014) que facilita la obtención de fuentes de información que respondan a un tema de investigación en específico, a partir de una revisión sistemática que proporciona rigurosidad para identificar artículos relevantes, de manera que se pueda abordar y sintetizar críticamente la revisión bibliográfica.

Realizamos una búsqueda en las bases de datos de Google Scholar, SpringerLink, IEEE Xplore y ScienceDirect debido a que son bases de datos de búsquedas bibliográficas más accesibles, frecuentes y fáciles de utilizar, y que además presentan mayor probabilidad de proveer información confiable; considerando criterios de filtro y selección de documentos en español, inglés y portugués que garanticen calidad en los resultados. Los artículos seleccionados han sido publicados en revistas indexadas a Latindex Catálogo, Scielo y principalmente Scopus. Se excluyeron documentos considerados literatura gris y aquellos que superasen los cinco años de antigüedad (2016 hasta el 2020) sumando un total de 48 artículos.

Parte de los documentos seleccionados fueron proporcionados por los servicios del Consejo Nacional de Ciencia, Tecnología e Innovación Tecnológica (CONCYTEC) del Estado Peruano, obteniendo información desde base de datos de pago. Posteriormente, a partir de los resultados de búsqueda, filtrado y selección de documentos se gestionó la información haciendo uso del gestor bibliográfico Mendeley para la sistematización de la información y la elaboración de citas y referencias, garantizando el cumplimento de criterios bibliográficos y editoriales.

\section{RESULTADOS DE LA REVISIÓN}

Los estudiantes del nivel superior necesitan incorporar como parte de sus competencias, durante su formación universitaria, el uso de herramientas tecnológicas para facilitar el proceso continuo de aprendizaje basado en la búsqueda de información; he ahí la importancia de reconocer estas herramientas como estrategias innovadoras en la educación (Molinero Bárcenas y Chávez Morales, 2019).

Para Vargas-Murillo (2019) los programas computacionales utilitarios y los referenciadores bibliográficos permiten gestionar la información y promover el uso continuo de recursos y herramientas digitales en el proceso investigativo.

Por otra parte, según Caraballoso Granado, Romero Romero y Castro Rodríguez (2019) las universidades afrontan la necesidad de mantener, generar o transformar entornos físicos o virtuales que permitan la constante generación de conocimientos, por lo cual los procesos de gestión de la información son recursos importantes para alcanzar los estándares de competencias exigidas por la sociedad.

En Perú, las universidades formulan reglamentos de investigación donde abordan criterios editoriales y bibliográficos que deben cumplirse; sin embargo, las evidencias de la presente revisión mostradas por Guillen-Valle et al. (2018); Barja-Ore et al. (2019) y Perdomo et al. (2020) señalan que los tesistas universitarios tienen deficiencias en el uso de referenciadores bibliográficos para abordar eficazmente el proceso de gestión de la información científica, siendo estos factores determinantes de la baja calidad de los resultados de las investigaciones.

Es ineludible reconocer que la adopción de los referenciadores bibliográficos implica contar con conocimientos básicos de informática; pues como mencionamos, la deficiencia de su uso, obliga en su mayoría a buscar páginas web de citación y referenciación que luego se realiza de manera manual como parte de la gestión de la información, generando consigo, según Guillen-Valle et al. (2018) la obstaculización de la recopilación necesaria de información científica para abordar el proceso investigativo.

Los resultados del desarrollo de la revisión se organizaron de acuerdo a las características, funciones, aspectos y estudios principales de los referenciadores bibliográficos que demuestren su importancia en la formación investigativa 
de los tesistas universitarios para gestionar la información científica con el objetivo de generar nuevos conocimientos que a su vez garanticen la calidad de los productos resultantes de sus investigaciones académicas.

\section{4 ¿QUÉ SON LOS REFERENCIADORES BIBLIOGRÁFICOS?}

De acuerdo a Escobar Fonseca y Hallo (2019) los referenciadores bibliográficos surgen como apoyo para dar solución a los problemas más comunes en la investigación, los cuales radican en la deficiencia de la gestión de la información científica.

Por ese motivo Murphree, White y Rochen Renner (2018) refieren que son herramientas estratégicas en el proceso investigativo que permiten gestionar la información a partir de la recopilación, almacenamiento y organización de la bibliografía, minimizando los esfuerzos del investigador para dar formato a las investigaciones en cuanto a estilos o normas de citas y referencias estandarizados, permitiendo dar valor agregado a los resultados de las investigaciones.

Según Gallegos, Peralta y Guerrero (2017) estas herramientas mantienen el orden de las fuentes de información consignadas como bibliografía en trabajos de investigación evitando errores de trascripción y facilitando la elaboración de citas y referencias bibliográficas de manera lógica y dinámica. Por lo tanto, se consideran como apoyo para garantizar la calidad bibliográfica y editorial en la elaboración de proyectos de investigación, informes de tesis, artículos científicos, entre otros, proporcionando ahorro de tiempo e impidiendo posibles fallos en el proceso de redacción.

\section{ESTUDIO Y DESCRIPCIÓN DE REFERENCIADORES BIBLIOGRÁFICOS}

Los referenciadores bibliográficos difieren respecto a determinadas características (Murphree, White y Rochen Renner, 2018), siendo tema de estudio que pretende determinar cuáles son los más funcionales y utilizados, así como cuáles proporcionan mayor eficiencia en las gestión de la información científica.

Investigaciones realizadas por Kratochvíl (2017); Santharooba y Lavanya (2018); Berry et al. (2020) y Fryš y Kovářová (2020) establecen comparaciones de sus funcionalidades o características con el objetivo de evaluar y establecer la precisión en la elaboración automática de citas y referencias según múltiples estilos o normas bibliográficas, así como aspectos de almacenamiento, soporte, costos y recursos.

Como ejemplo, mostramos los resultados más resaltantes del estudio de Berry et al. (2020) quienes encuestaron a 70 individuos entre estudiantes, profesores y personal de la Universidad Estatal de Kennesaw (EE.UU.), obteniendo los siguientes datos presentados a continuación (Tabla I y II).

\begin{tabular}{lccc}
\hline Gestor & Facultad (\%) & Estudiantes (\%) & Personal (\%) \\
\hline BibTeX & 37 & 6 & 14 \\
EndNote desktop & 78 & 74 & 57 \\
EndNote online & 48 & 42 & 14 \\
JabRef & 4 & 3 & 0 \\
Mendeley & 22 & 23 & 14 \\
Microsoft & 7 & 16 & 43 \\
ReadCube & 0 & 3 & 0 \\
Refworks & 19 & 3 & 0 \\
Zotero & 48 & 6 & 14 \\
\hline
\end{tabular}

Tabla I. Referenciadores bibliográficos más utilizados. Fuente: Traducido de Berry et al. (2020).

\begin{tabular}{lcccc}
\hline Respuestas & Facultad \% & Personal \% & Estudiantes \% & Total \% \\
\hline Insertar citas en un documento & 43 & 8 & 36 & 88 \\
Exportar citas desde bases de datos al programa de & 32 & 4 & 3 & 61 \\
gestión bibliográfica & 31 & 6 & 31 & 67 \\
Organización de la información & 13 & 3 & 13 & 28 \\
Anotar referencias o archivos PDFs & 31 & 6 & 31 & 67 \\
Compartir referencias con otros & 18 & 6 & 19 & 43 \\
\hline
\end{tabular}

Tabla II. Características más importantes. Fuente: Traducido de Berry et al. (2020). 
Según los datos proporcionados se reconoce la peculiaridad en el uso del referenciador bibliográfico EndNote desktop, producto de la familiaridad de sus usuarios con la funcionalidad del software (facilidad de uso), y esto se debe a que dicho gestor es patrocinado por la misma biblioteca de la institución.

Asimismo, se identifica que entre las funcionalidades más importantes que brindan los referenciadores bibliográficos son la inserción de citas y referencias en los documentos de investigación, además de proporcionar viabilidad en la organización de la información y compartir bibliografías entre equipos de investigadores.

Estos resultados expresan que la diversidad de referenciadores bibliográficos, o también denominados, sistemas o software de gestión de citas, presentan funcionalidades propias que conllevan a los usuarios a depender de sus fortalezas para ser adoptadas según diferentes necesidades.

A continuación, describimos los tres gestores de referencias bibliográficas más utilizadas a nivel iberoamericano según estudios de Antúnez Sánchez y Veytia Bucheli (2020) y complementados con los resultados de Berry et al. (2020).

\section{Mendeley (www.mendeley.com)}

Mendeley es un administrador de referencias bibliográficas lanzado en 2008 por estudiantes de doctorado alemanes, posteriormente (2013) fue adquirido por la editorial Elsevier. Funciona como red social académica de investigadores y permite organizar trabajos de investigación científica, compartir resultados y sobre todo gestionar información. Está disponible en múltiples plataformas como Windows, Mac y Android, y además funciona como programa basado en la web para promover el interés y discusión sobre temas de investigación. La plataforma cuenta con 2 gigabytes de espacio de almacenamiento gratuito y adicionalmente disponible por tarifas (Elston, 2019).

\section{EndNote (www.endnote.com)}

EndNote es un sistema de gestión de citas desarrollado por Clarivate Analytics. Entre sus principales características resalta la posibilidad de compilar y compartir materiales de investigación y bibliografía minimizando los esfuerzos de colaboración entre investigadores, de modo que las citas y referencias son accesibles desde diferentes espacios. Los usuarios suscritos en la plataforma pueden generar informes de citas para diferentes grupos seleccionados en una biblioteca de notas, proporcionando estadísticas que desglosan por años y número de veces que se citaron los artículos en la biblioteca de EndNote. La accesibilidad a la plataforma se puede realizar en línea, aplicaciones de escritorio e iPad (Hupe, 2019).

\section{Zotero (www.zotero.org)}

Zotero fue lanzado en el año 2006 por la Universidad George Mason de Estados Unidos como software bibliográfico para capturar información sobre temas de investigación, crear citas y referencias y administrar la literatura recopilada. Permite almacenar y organizar bibliografías en base de datos personales para posteriormente generar citas y referencias de acuerdo a diferentes estilos bibliográficos. Entre sus características resalta que la herramienta es de código abierto por lo que su adopción genera rentabilidad, disponibilidad de la fuente de código y facilidad de modificación. Asimismo, destaca por detectar metadatos disponibles en sitios webs, facilitando la obtención de información (Kumar Ray y Ramesh, 2017).

\section{FUNCIONES POTENCIALES DE LOS REFERENCIADORES BIBLIOGRÁFICOS}

Acá, consolidamos las funcionalidades en común de los referenciadores bibliográficos que consideramos potencialmente útiles para realizar la gestión de la información científica en la elaboración de trabajos investigativos por tesistas investigadores.

\subsection{Organización de la información}

Para establecer el significado de la organización de la información nos basamos en Hernández Quintana (2017) quien señala que dicho término se justifica en la sistematización enciclopédica y metodológica de las ciencias, ya que trabajar con ello no es abstracto, sino registrado y estructurado. Ahora, en relación a la investigación científica y considerando como principal proceso la gestión de la información, Bouza Betancourt, Couto Torres y Sosa Pérez (2017) sostienen que uno de los problemas principales que afrontan los investigadores es organizar la información debido al exceso de la disponibilidad de los mismos en diferentes fuentes bibliográficos (físicos o digitales).

La información recopilada carece de sistematización, pues en su mayoría los investigadores realizan el proceso de manera manual a través carpetas u hojas de cálculo, siendo estos los factores directos de la deficiente organización de la información científica y calidad de la investigación (Gallegos, Peralta y Guerrero, 2017). Frente a ello, la 
disponibilidad de los referenciadores bibliográficos surge como recurso necesario e indispensable para los tesistas universitarios, puesto que brindan herramientas que permiten recopilar, almacenar, organizar y sobre todo gestionar la información consolidada como utilizable en el proceso investigativo.

\subsection{Citación y referenciación bibliográfica}

Se han llevado a cabo diversas investigaciones para evaluar la calidad de las tesis de grado en las universidades desde diferentes perspectivas como calidad metodológica, estadística, resumen, referencias bibliográficas, etc.; así, los estudios realizados por Valiente Sandó, Salazar Salazar y Domínguez Rodríguez (2018); Barja-Ore et al. (2019) y Perdomo et al. (2020) identifican deficiencias en la calidad de las investigaciones, como la inadecuada elaboración de citas y referencias bibliográficas, las cuales no se rigen ni aplican estilos bibliográficos producto del desconocimiento de las normas de citación y referenciación.

La citas y referencias bibliográficas permiten a los investigadores ser éticos; ya que, según Yang, Lee y Choi (2016) en los últimos tiempos se ha considerado a las citas bibliográficas parte del estudio de las ciencias bibliométricas, el cual permite estudiar la productiva de la investigación, así como el impacto y calidad de las mismas en cuanto al número de veces que la información fue empleada por brindar confiabilidad y tendencias de su uso como recurso de información (Repiso, Moreno-Delgado y Aguaded, 2020); por ende, consolidar conocimientos en la elaboración de citas y referencias bibliográficas adecuadamente en documentos científicos es de suma importancia para los investigadores en general.

A propósito de consolidar la revisión realizada, el estudio realizado por Habib y Afzal (2019) asegura que la sobrecarga de información en múltiples plataformas dificulta a los investigadores en el acceso a fuentes de información relevantes, generando, al mismo tiempo, conflictos para citar información como parte integral de la investigación académica (Färber y Jatowt, 2020). Se suma a esto, el bajo dominio de las normas y/o estilos bibliográficos orientados y estandarizados según diferentes ramas de las ciencias (Pérez Infante y Bayés Cáceres, 2017) siendo factores determinantes de la baja calidad de la investigación.

Los referenciadores bibliográficos brindan soporte tecnológico para la elaboración automática de citas y referencias bibliográficas mediante la cosecha de metadatos (Titulo, Autores, Revista, Año, Volumen, Ejemplar, Paginas, Url o Doi) que posteriormente tienen que ser arbitrados por los investigadores con la finalidad de garantizar la correcta citación y referenciación en documentos científicos. Esto permite que los tesistas universitarios se enfoquen más al proceso investigativo, ya que se minimiza la presión en dedicar mayor tiempo en la elaboración adecuada de las citas y referencias bibliográficas. Esto representa la solución al problema planteado.

\subsection{Colaboración entre investigadores}

El constante avance de las TIC's de la mano del fenómeno de la digitalización ha ocasionado cambios fructíferos en los sistemas de elaboración y distribución de la información, y consecuentemente la forma de entender la investigación (Flores-Vivar y Zaharía, 2019). Así, la influencia de las tecnologías y la sociedad actual en el proceso de educación superior permite a docentes y estudiantes desarrollar pensamientos críticos, flexibles e innovadores para adaptarse a nuevos roles, mejorar la alfabetización informacional y sobre todo emplear habilidades de procesamiento de la gestión científica (Antúnez Sánchez y Veytia Bucheli, 2020).

Flores-Vivar y Zaharía (2019) señalan que debido a la arquitectura tecno-comunicativa en la investigación han surgido las redes sociales científicas y académicas con el objetivo de crear canales de comunicación que satisfaga la colaboración entre investigadores. Se plantea entonces la necesidad de desarrollar investigaciones académicas con la participación tanto de catedráticos como autoridades y estudiantes, además de prestar mayor atención a la producción científica como estrategia para fomentar cultura investigativa y crecimiento profesional (Auza-Santiváñez, SantiváñezCabezas y Dorta-Contreras, 2020).

El rol que cumplen los referenciadores bibliográficas es sustancial pues permiten establecer relaciones comunicativas para facilitar la colaboración entre investigadores con el fin de abordar una temática o producción académica (Grijalva et al., 2019), pues como lo describimos, las funcionalidades de estas herramientas para organizar los trabajos académicos grupales y el intercambio de materiales de investigación resultan favorables para los tesistas universitarios.

\subsection{Limitaciones de adopción de los referenciadores bibliográficos}

Según Murphree, White y Rochen Renner (2018) la experiencia de los investigadores con los referenciadores bibliográficos puede variar de acuerdo a diferentes contextos, usuarios, usos y evolución en el tiempo debido a las fortalezas, debilidades y características propias de cada herramienta, siendo estos factores los que limitan su adopción. Además, consideramos entre las limitantes el bajo compromiso de las universidades en enfocar esfuerzos para la 
incorporación de herramientas tecnológicas como los referenciadores bibliográficos que permitan generar conocimiento en los tesista sobre la adecuada gestión de la información científica, de manera que se ve reflejado la inconveniencia y desinterés en la enseñanza de los docentes (Berry et al., 2020) producto del desconocimiento en el uso de las TIC's.

\section{CONCLUSIONES}

En este artículo presentamos una revisión sistemática sobre la importancia de los referenciadores bibliográficos en la gestión de la información científica en tesistas universitarios. La metodología de revisión que adoptamos nos permitió realizar una visión completa y actual de los temas relacionados al objetivo del estudio, al mismo tiempo validar los resultados a partir de las fases establecidas para abordar una revisión sistemática fundamentada.

En el desarrollo de la revisión, resaltamos que los tesistas universitarios tienen dificultades para gestionar la información científica en el proceso investigativo académico, que se incrementa por la constante evolución tecnológica de medios o plataformas para acceder, recopilar, administrar, organizar y analizar la información, que tienen que ser utilizados éticamente en los documentos científicos reconociendo el aporte de otros a través de citas y referencias bibliográficas.

Por lo tanto, adoptar el uso de los referenciadores bibliográficos resulta de suma importancia, pues sus funcionalidades para organizar la información, gestionar citas y referencias bibliográficas y permitir la colaboración entre equipos de investigadores a través de redes de comunicación científica, son recursos tecnológicos estratégicos para gestionar eficientemente la información científica, con ello garantizar la calidad bibliográfica, editorial, académica y científica en los resultados de las investigaciones desarrolladas por tesistas universitarios.

\section{BIBLIOGRAFÍA}

ANTÚNEZ SÁNCHEZ, A.G. y VEYTIA BUCHELI, M.G. Desarrollo de competencias investigativas y uso de herramientas tecnológicas en la gestión de información. Revista Conrado, 2020, vol. 16, n 72, p. 96-102.

ATAMARI-ANAHUI, N.; SUCASACA-RODRÍGUEZ, C. y MARROQUIN-SANTA CRUZ, J.A. Publicación científica de asesores de tesis de pregrado en una escuela de medicina de Cusco, Perú. Investigación en Educación Médica, 2016, vol. 5, n 20, p. 279-280. http://dx.doi.org/10.1016/j.riem.2016.05.002.

AUZA-SANTIVÁÑEZ, J.C.; SANTIVÁÑEZ-CABEZAS, M.V. y DORTA-CONTRERAS, A.J. Análisis de la producción científica y la colaboración internacional boliviana indexada en Scopus entre 1996-2018. Revista Cubana de Investigaciones Biomédicas, 2020, vol. 39, $\mathrm{n}^{\circ} 3$, p. 1-16.

BARJA-ORE, J.; OTOYA-PETIT, Ó.; VEGA-GONZALES, E.O.; MORENO-GUTIERREZ, N. y LOLI-PONCE, R.A. Actitudes hacia la investigación de internos de obstetricia rotantes en un hospital de Lima - Perú. Revista de la Facultad de Medicina Humana, 2019, vol. 19, n 4, p. 53-59. http://dx.doi.org/10.25176/RFMH.v19i4.2341.

BERRY, T.U.; MILEWSKI, S.D.; SEXTON, M.C.; SHARP, A.L. y WILLIAMSON, J.M. Understanding user needs for citation management program support. Public Services Quarterly, 2020, vol. 16, n ${ }^{\circ}$ 2, p. 71-82. http://dx.doi.org/10.1080/15228959.2019.1687070.

BOUZA BETANCOURT, O.; COUTO TORRES, D. y SOSA PÉREZ, N. de la C. Evaluación del estado de la gestión de la información científica y tecnológica: dimensiones e indicadores. Revista Cubana de Informacion en Ciencias de la Salud, 2017, vol. 28, n 4, p. 1-17.

CANTÍN, M. y CHUHUAICURA, P. Estilos de citas y referencias bibliográficas en revistas odontológicas SciELO: Un elemento crítico de la escritura científica. International journal of odontostomatology, 2016, vol. 10 , $\mathrm{n}^{\circ} 2$, p. 349 357. http://dx.doi.org/10.4067/S0718-381X2016000200024.

CARABALLOSO GRANADO, K.; ROMERO ROMERO, O. y CASTRO RODRÍGUEZ, M.E. Uso de herramientas de gestión de información en bibliotecas universitarias, efecto en la actividad científica: estudio de caso. Revista Interamericana de Bibliotecología, 2019, vol. 42, $\mathrm{n}^{\circ}$ 2, p. 141-157. http://dx.doi.org/10.17533/udea.rib.v42n2a03.

CAYO-ROJAS, F.C. y AGRAMONTE-ROSELL, R. de la C. Estrategias para la formación de una Sociedad Científica de Estudiantes de Odontología en Perú. Educación Médica, 2020, p. 1-2. http://dx.doi.org/10.1016/j.edumed.2020.05.004.

DAL SASSO MENDES, K.; DE CAMPOS PEREIRA SILVEIRA, R.C. y GALVÃO, C.M. Use of the bibliographic reference manager in the selection of primary studies in integrative reviews. Texto \& Contexto - Enfermagem, 2019, vol. 28, n e20170204, p. 1-13. http://dx.doi.org/10.1590/1980-265X-TCE-2017-0204 1/13.

DÍEZ, J.; GULLÓN, P. y FERNÁNDEZ, E. El estilo bibliográfico de Gaceta Sanitaria en los gestores de referencias bibliográficas. Gaceta Sanitaria, 2019, vol. 33, n 3, p. 300-301. http://dx.doi.org/10.1016/j.gaceta.2018.06.012.

ELSTON, D.M. Mendeley. Journal of the American Academy of Dermatology, 2019, vol. 81, n 5, p. 1071. http://dx.doi.org/10.1016/j.jaad.2019.06.1291. 
ESCOBAR FONSECA, M.F. y HALLO, M. 2019. Integration of Tools for Handling Open Access Scientific Articles. 2019 International Conference on Information Systems and Software Technologies (ICI2ST). S.1.: IEEE, http://dx.doi.org/10.1109/ICI2ST.2019.00012.

FÄRBER, M. y JATOWT, A. Citation recommendation: approaches and datasets. International Journal on Digital Libraries, 2020, vol. 21, n 4, p. 375-405. http://dx.doi.org/10.1007/s00799-020-00288-2.

FERNÁNDEZ IZQUIERDO, F. Una aproximación a los instrumentos metodológicos digitales: los gestores bibliográficos. Ayer: Revista de Historia Contemporánea, 2018, vol. 110, n² 2, p. 51-82.

FLORES-VIVAR, J.M. y ZAHARÍA, A.M. Las redes sociales académicas en la difusión de la producción científica. Estudio de caso: Academia y Mendeley. Estudios sobre el Mensaje Periodístico, 2019, vol. 25, n 3, p. $1379-1402$. http://dx.doi.org/10.5209/esmp.66993.

FRYŠ, J. y KOVÁŘ OVÁ, P. Testování funkcí citačních manažerů: komparace Citace PRO, Citavi, EndNote, Mendeley a Zotero. ProInflow, 2020, vol. 12, n 1, p. 29-60. http://dx.doi.org/10.5817/ProIn2020-1-3.

GALLEGOS, M.C.; PERALTA, C.A. y GUERRERO, W.M. Utilidad de los gestores bibliográficos en la organización de la información para fines investigativos. Formación Universitaria, 2017, vol. 10, $\mathrm{n}^{\circ}$ 5, p. 77-85. http://dx.doi.org/10.4067/S0718-50062017000500009.

GARCÍA VALDÉS, D. Gestión y uso de la información científica técnica de las carreras contabilidad, derecho y economía en la Universidad de Cienfuegos. Revista Universidad y Sociedad, 2016, vol. 8, n 2, p. 153-159.

GRIJALVA, P.K.; CORNEJO, G.E.; GÓMEZ, R.R.; REAL, K.P. y FERNÁNDEZ, A. Herramientas colaborativas para revisiones sistemáticas. Revista Espacios, 2019, vol. 40, n 45 .

GUILLEN-VALLE, O.R.; GONDO-MINAMI, R.; CERNA-VENTURA, B.F.; MARTÍNEZ， E. y PACHECOSAAVEDRA, A. Gestor de referencia como herramienta en la reducción de similitudes en trabajos de investigación. Revista Peruana de Salud Pública y Comunitaria, 2018, vol. 1, n 2, p. 83-84.

HABIB, R. y AFZAL, M.T. Sections-based bibliographic coupling for research paper recommendation. Scientometrics, 2019, vol. 119, n 2, p. 643-656. http://dx.doi.org/10.1007/s11192-019-03053-8.

HERNÁNDEZ QUINTANA, A.R. Resilencia de la organización de la información en la era de la posverdad. $A L C A N C E$ Revista Cubana de Información y Comunicación, 2017, vol. 6, n 14, p. 47-59.

HUPE, M. EndNote X9. Journal of Electronic Resources in Medical Libraries, 2019, vol. 16, n 3-4, p. 117-119. http://dx.doi.org/10.1080/15424065.2019.1691963.

KRATOCHVÍL, J. Comparison of the Accuracy of Bibliographical References Generated for Medical Citation Styles by EndNote, Mendeley, RefWorks and Zotero. The Journal of Academic Librarianship, 2017, vol. 43, n 1, p. 5766. http://dx.doi.org/10.1016/j.acalib.2016.09.001.

KUMAR RAY, A. y RAMESH, D. Zotero: Open Source Citation Management Tool for Researchers. International Journal of Library and Information Studies, 2017, vol. 7, n 3, p. 238-245.

LÓPEZ PADRÓN, A.; AMAURIS LAURENCIO, L.; ZAMBRANO ACOSTA, J.M. y QUIROZ FERNÁNDEZ, L.S. Principales problemas identificados en tesis de doctorado en Ciencias de la Educación: una sistematización de experiencias. Revista Cubana de Educación Superior, 2019, vol. 38, n 2.

MARTÍN, S.G. y LAFUENTE, V. Referencias bibliográficas: Indicadores para su evaluación en trabajos científicos. Investigacion Bibliotecologica, 2017, vol. $\quad 31, \quad \mathrm{n}^{\circ} \quad 71, \quad$ p. $\quad 151-180$. http://dx.doi.org/10.22201/iibi.0187358xp.2017.71.57814.

MEDINA MARTÍNEZ, O.D. et al. Sistema de información para la gestión de la producción científica de la Universidad de Ciencias Médicas de Cienfuegos. Medisur, 2020, vol. 17, n 6, p. 764-770.

MILEWSKI, S.D. y WILLIAMSON, J.M. For Your Enrichment: Developing a Reflective Practice Template for Citation Management Software Instruction. Reference \& User Services Quarterly, 2017, vol. 57, $\mathrm{n}^{\circ}$ 1, p. 6-11. http://dx.doi.org/10.5860/rusq.57.1.6435.

MOLINERO BÁRCENAS, M. del C. y CHÁVEZ MORALES, U. Herramientas tecnológicas en el proceso de enseñanza-aprendizaje en estudiantes de educación superior. RIDE Revista Iberoamericana para la Investigación y el Desarrollo Educativo, 2019, vol. 10, n 19. http://dx.doi.org/10.23913/ride.v10i19.494.

MURPHREE, L.; WHITE, M. y ROCHEN RENNER, B. Reference Managers that Support Collaborative Research: Dreaming of the Perfect Fit. Medical Reference Services Quarterly, 2018, vol. 37, n ${ }^{\circ}$ 3, p. $219-233$. http://dx.doi.org/10.1080/02763869.2018.1477706.

PERDOMO, B. et al. Calidad de las tesis de pregrado en universidades peruanas. Revista Espacios, 2020, vol. 41, $\mathrm{n}^{\circ}$ 02.

PÉREZ INFANTE, Y. y BAYÉS CÁCERES, E. Utilidad de una guía metodológica para la elaboración de proyectos de investigación. Medisan, 2017, vol. 21, n 2, p. 237-242.

REPISO, R.; MORENO-DELGADO, A. y AGUADED, I. Factors affecting the frequency of citation of an article. Iberoamerican Journal of Science Measurement and Communication, 2020, vol. 1, $\mathrm{n}^{\circ}$ 1, $\mathrm{p}$. 1-6. http://dx.doi.org/10.47909/ijsmc.08. 
REYES PÉREZ, J.J.; CÁRDENAS ZEA, M.P. y GAVILÁNEZ BUÑAY, T.C. Desarrollo de competencias investigativas medidas por tecnologías en estudiantes de la carrera de agronomía. Revista Conrado, 2020, vol. 16, $\mathrm{n}^{\circ} 73$, p. 108-113.

RIESENBERG, L.A. y JUSTICE, E.M. Revisión sistemática de la bibliografía (parte 1). Nursing, 2014, vol. 31, nº 6, p. 61-64. http://dx.doi.org/10.1016/j.nursi.2014.12.019.

RIVERA ECHEGARAY, L.A.; PIRRO LOZANO, M.M.; VARGAS ESPINOZA, J.L.; BOLLET RAMÍREZ, F. y FLORES SALDAÑA, L.E. Gestión de la información científica con repositorios digitales y formación investigativa de los estudiantes de la Facultad de Ingenieria Agroindustrial Universidad Nacional Intercultural de la Amazonía 2015. Repositorio de Revistas de la Universidad Privada de Pucallpa, 2017, vol. 2, n 4, p. 9-12. http://dx.doi.org/10.37292/riccva.v2i04.77.

RIVERA OBREGÓN, M.L.; LÓPEZ LÓPEZ, M.L.; SIU QUEVEDO, M.E.; ZEA GORDILLO, R.E. y ZAPATA RIVERA, I.L. 2018. La competencia de la gestión de la información científica en los programas de posgrado profesionalizantes: la tutoría como eje contribuidor a su desarrollo. Congreso Internacional de Investigacion Academia Journals. S.1.: s.n.

RODRÍGUEZ PICORNELL, Z. y DEL POZO RODRÍGUEZ, P.P. Gestión de la información científica en la Universidad Agraria de la Habana. Ciencias de la Información, 2019, vol. 50, n 3, p. 17-23.

ROJAS BETANCUR, H.M. y MÉNDEZ VILLAMIZAR, R. Procesos de formación en investigación en la Universidad: ¿Qué le queda a los estudiantes? Sophia, 2017, vol. 13, n 2, p. 53-69. http://dx.doi.org/10.18634/sophiaj.13v.2i.261.

SANTHAROOBA, S. y LAVANYA, J. 2018. A Comparative Analysis of Reference Managers: EndNote and Mendeley. International Conference of University Librarians Association of Sri Lanka. S.1.: s.n.

SEMELER, A.R. y PINTO, A.L. Data librarianship as a field study. Transinformação, 2020, vol. 32, n e200034, p. 112. http://dx.doi.org/10.1590/2318-0889202032e200034.

VALIENTE SANDÓ, P.; SALAZAR SALAZAR, M. y DOMÍNGUEZ RODRÍGUEZ, L. La calidad de las tesis doctorales en ciencias pedagógicas: visión desde una muestra de informes de oponencia. Revista Cubana de Educación Superior, 2018, vol. 37, n³ , p. 41-58.

VALLES-CORAL, M.A. Modelo de gestión de la investigación para incrementar la producción científica de los docentes universitarios del Perú. Revista de Investigación, Desarrollo e Innovación, 2019, vol. 10, n 1, p. 67-78. http://dx.doi.org/10.19053/20278306.v10.n1.2019.10012.

VALLES-CORAL, M.A.; RIASCOS-ARMAS, J.O. y HERNÁNDEZ-TORRES, E.A. Gestión de la identidad digital del investigador y su efecto en el ranking webométrico de una universidad amazónica peruana. Revista Cubana de Informacion en Ciencias de la Salud, 2020, vol. 31, n 2, p. 1-17. http://dx.doi.org/10.36512/rcics.v31i2.1406.g897.

VARGAS-MURILLO, G. Competencias digitales y su integración con herramientas tecnológicas en educación superior. Revista Cuadernos, 2019, vol. 60, n 1, p. 88-94.

YANG, K.; LEE, J. y CHOI, W. Publication and citation patterns of Korean LIS research by subject areas. Malaysian Journal of Library \& Information Science, 2016, vol. 21, n 2, p. 67-81. http://dx.doi.org/10.22452/mjlis.vol21 no2.5. 